JOURNAL OF

APPLIED

CRYSTALLOGRAPHY

ISSN 1600-5767

\section{Direct protein crystallization on ultrathin membranes for diffraction measurements at $\mathrm{X}$-ray free-electron lasers}

\author{
Nadia Opara, ${ }^{a, b, c *}$ Isabelle Martiel, ${ }^{a}$ Stefan A. Arnold, ${ }^{b, c}$ Thomas Braun, ${ }^{b, c}$ Henning \\ Stahlberg, ${ }^{b, c}$ Mikako Makita, ${ }^{a}$ Christian David ${ }^{a}$ and Celestino Padeste ${ }^{a, c}$
}

Received 19 December 2016

Accepted 18 April 2017

Edited by A. Barty, DESY, Hamburg, Germany

Keywords: in situ crystallization; nanomembrane chips; serial femtosecond crystallography; time-resolved measurement; $\mathrm{X}$-ray free-electron lasers.

PDB reference: lysozyme, 5 ne0

Supporting information: this article has supporting information at journals.iucr.org/j

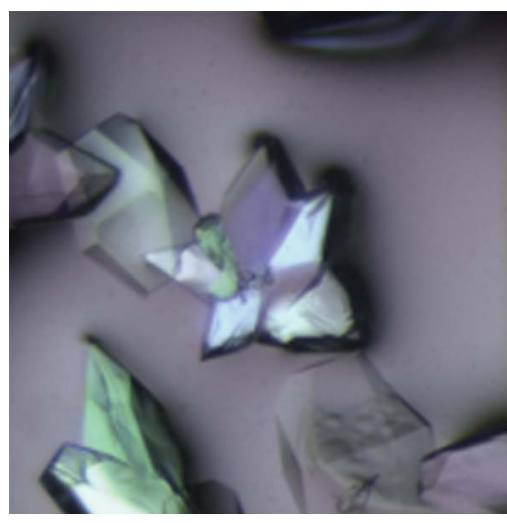

C 2017 International Union of Crystallography
aPaul Scherrer Institute (PSI), 5232 Villigen, Switzerland, ${ }^{\mathbf{b}}$ Center for Cellular Imaging and NanoAnalytics (C-CINA), Biozentrum, University of Basel, Basel 4058, Switzerland, and 'Swiss Nanoscience Institute, University of Basel, Basel 4056, Switzerland. *Correspondence e-mail: nadia.opara@psi.ch

A new era of protein crystallography started when X-ray free-electron lasers (XFELs) came into operation, as these provide an intense source of X-rays that facilitates data collection in the 'diffract-before-destroy' regime. In typical experiments, crystals sequentially delivered to the beam are exposed to X-rays and destroyed. Therefore, the novel approach of serial crystallography requires thousands of nearly identical samples. Currently applied sample-delivery methods, in particular liquid jets or drop-on-demand systems, suffer from significant sample consumption of the precious crystalline material. Direct protein microcrystal growth by the vapour diffusion technique inside arrays of nanolitre-sized wells is a method specifically tailored to crystallography at XFELs. The wells, with X-ray transparent $\mathrm{Si}_{3} \mathrm{~N}_{4}$ windows as bottoms, are fabricated in silicon chips. Their reduced dimensions can significantly decrease protein specimen consumption. Arrays provide crystalline samples positioned in an ordered way without the need to handle fragile crystals. The nucleation process inside these microfabricated cavities was optimized to provide high membrane coverage and a quasi-random crystal distribution. Tight sealing of the chips and protection of the crystals from dehydration were achieved, as confirmed by diffraction experiments at a protein crystallography beamline. Finally, the test samples were shown to be suitable for time-resolved measurements at an XFEL at femtosecond resolution.

\section{Introduction}

Detailed structural information on proteins and other biomacromolecules is crucial to understand biochemical processes and design new medicines. Several methods are routinely used to obtain such information, e.g. nuclear magnetic resonance (Billeter et al., 2008; Wüthrich, 1990; Marassi \& Opella, 1998), electron microscopy (EM) (Binshtein \& Ohi, 2015; Ruprecht \& Nield, 2001; Vinothkumar, 2015) and X-ray diffraction (Lamb et al., 2015; Moraes et al., 2014; Wlodawer et al., 2007). X-ray free-electron lasers (XFELs) are rapidly becoming an indispensable addition (Fromme, 2015). They provide femtosecond X-ray pulses of ultrahigh peak brilliance, which allows the detection of diffraction signals from crystals much smaller than those studied at synchrotron sources, i.e. even from nano- and twodimensional crystals (Falkner et al., 2005; Martin \& Zilm, 2003). This is made possible by outrunning radiation damage in the 'diffract-before-destroy' regime: compared with synchrotrons, a much higher dose is applied in a time shorter 
than that of typical X-ray induced molecular rearrangement or damage processes, and the diffraction signals arising from one single X-ray pulse are collected. However, when a pulse of the extremely intense radiation interacts with the sample, the crystal is ultimately damaged and no longer diffracts, which means that it cannot be used again. Thus, data collection has to be carried out in a serial approach where femtosecond diffraction experiments are performed on a large number of individual protein crystals (Schlichting, 2015).

Adequately prepared samples containing thousands of crystals, each of which might be smaller than $1 \mu \mathrm{m}$, have to be delivered to the beam in a short time. To this end, injectorbased methods for aqueous suspensions (Hunter \& Fromme, 2011) and lipidic cubic phase (LCP) (Weierstall et al., 2014) have been successfully applied to achieve this for both soluble and membrane proteins, in order to determine their structure. In particular, the liquid jets require large amounts of sample in order to obtain full and conclusive data sets, owing to the high flow rate. In an ideal case, when crystals are embedded in a slowly flowing viscous medium such as agarose, hyaluronic acid or LCP prior to injection, a flow rate of $160 \mathrm{nl} \mathrm{min}^{-1}$ is achieved, while typical flow rates for aqueous suspensions can be up to 100 times higher (Conrad et al., 2015; Sugahara et al., 2016).

Alternatively, samples are immobilized on solid supports. This 'fixed-target' approach has the advantage that it uses significantly less biological material and allows a visual preselection of target sites. In one of the first time-delay holography experiments with an XFEL, polystyrene spheres $140 \mathrm{~nm}$ in diameter were deposited on $20 \mathrm{~nm}$ silicon nitride $\left(\mathrm{Si}_{3} \mathrm{~N}_{4}\right)$ membranes present in a silicon chip backed by a multilayer-coated plane mirror to form a sandwich-like packaging, and used for femtosecond time-delay X-ray holography (Chapman et al., 2007). Since then, there have been numerous attempts to optimize the fixed-target approach. In particular, deterioration of the sample upon drying and the transparency of the support material have to be considered. In addition, protein crystals are very fragile. They are prone to losing quality owing to dehydration, and require preservation or waterproof protection. The methods used to maintain crystal quality while diffraction data are collected include sugar-embedding (e.g. using trehalose; Schebor et al., 2010), Paratone-N oil coverage (Hunter et al., 2014), vitrification (Sader et al., 2009), 'sandwich' enclosure (Coquelle et al., 2015) and wrapping in watertight graphene sheets (Wierman et al., 2013).

The microfabrication strategy used to obtain relatively transparent thin freestanding $\mathrm{Si}_{3} \mathrm{~N}_{4}$ membranes in silicon frames is well established and widely applied, e.g. as described by Gibb \& Ayub (2013). Windows of this type, in some cases with an octadecyltrichlorosilane coating, and windows of thin carbon film with a poly(methyl methacrylate) layer (Frank et al., 2014) were used for the collection of diffraction data of two-dimensional crystals of membrane proteins embedded in sugar (Pedrini et al., 2014). Zarrine-Afsar and co-workers deposited concentrated suspensions of lysozyme and ferritin three-dimensional crystals onto $\mathrm{Si}_{3} \mathrm{~N}_{4}$ membranes for XFEL experiments (Zarrine-Afsar et al., 2012). Microfabricated silicon chips combined with a Mylar foil backing were also described as suitable for visual inspection and high-speed spectroscopy mapping for red myoglobin crystals (Oghbaey et al., 2016). Development towards full automation of serial data collection has been shown with X-CHIP designs (Kisselman et al., 2011). Goniometer-based serial femtosecond crystallography on polymeric meshes and grids has also been reported (Cohen et al., 2014) as an example of a helical or raster-scanning data-collection strategy avoiding XFEL exposure of a previously exposed sample area.

Recently, supports that allow the fixed-target X-ray analysis of three-dimensional microcrystals deposited in small wells (Roedig et al., 2015; Baxter et al., 2016; Murray et al., 2015) or enclosed in between thin polymer films (Axford et al., 2016) were developed. A microfluidic system that allows microcrystals grown elsewhere to be harvested and examined directly in an array of hydrodynamic traps is also available and provides an efficient way of capturing individual crystals at defined positions (Lyubimov et al., 2015).

Large three-dimensional protein crystals can be obtained by various standard methods, e.g. crystallization in gels (Matsumura et al., 2011), under microgravity (McPherson \& Lawrence, 2015) or by seeding (Shimizu et al., 2009). The growth of crystals directly on a support, which are subsequently placed in the XFEL beam, would be particularly attractive for two-dimensional protein crystals, as the quality of the crystals can deteriorate when they are handled. Such samples have been employed for atomic force microscopy and EM studies. For example, two-dimensional streptavidin crystals were grown on mica surfaces covered by phospholipids partially modified by biotin (Lou et al., 2008); the morphology of the crystals obtained depended on the $\mathrm{pH}$ value of the mother liquid. Further, large (approximately $5 \mu \mathrm{m}$ ) twodimensional crystals and ultrathin three-dimensional crystals of the $\mathrm{H}^{+}$-ATPase found in the plasma membrane of the red bread mould Neurospora crassa were grown directly on the surface of a carbon-film supported gold-plated copper EM grid (Auer et al., 1999).

Here, we report the direct crystallization of proteins on $\mathrm{Si}_{3} \mathrm{~N}_{4}$ nanomembranes for fixed-target XFEL experiments. The silicon chip design and microfabrication steps that can be used in combination with nanolitre-volume liquid-handling systems are described. The growth of crystals by vapour diffusion methods on $\mathrm{Si}_{3} \mathrm{~N}_{4}$ membranes and the use of a sealed asymmetric sandwich system developed to prevent crystals from drying before being probed by the XFEL beam are demonstrated. The described in situ crystal growth (as an example of the fixed-target approach) is especially useful when large quantities of similar crystals are required, e.g. for pump-probe experiments at XFELs. One specific aim of the crystallization experiments was to provide many crystals that are large enough to be hit in a split-delay X-ray optical setup for pump-probe experiments (David et al., 2015) by two sets of X-ray beams (pump-probe and reference) that illuminate, respectively, pumped and reference areas separated by approximately $70 \mu \mathrm{m}$. 


\section{Experimental details}

\subsection{Microfabrication}

Single-crystal silicon $\langle 100\rangle$ wafers, $240-260 \mu \mathrm{m}$ in thickness and $100 \mathrm{~mm}$ in diameter, obtained by the Czochralski process (Si-Mat Silicon Materials, Germany) and polished on both sides, were covered with a $250 \mathrm{~nm}$ thick layer of low-stress amorphous $\mathrm{Si}_{3} \mathrm{~N}_{4}$ grown by low-pressure chemical vapour deposition (CVD) (Centre de MicroNanoTechnologie, EPFL, Switzerland). Subsequently, a positive tone resist (S-1813, Microposit, USA) was spin-coated onto the $\mathrm{Si}_{3} \mathrm{~N}_{4}$ layer on both sides of the wafer using a spin speed of $4000 \mathrm{r} \mathrm{min}^{-1}$ for $40 \mathrm{~s}$ and an initial ramp of $400 \mathrm{r} \mathrm{min}^{-1} \mathrm{~s}^{-1}$. The deposited layer was soft-baked on a hot plate at $388 \mathrm{~K}$ for $90 \mathrm{~s}$. The resist on one side of the wafer was then patterned [Fig. 1(a), part (i)] with the template for $\mathrm{Si}_{3} \mathrm{~N}_{4}$ windows by a photolithography process (see $\$ 3.1$, and Fig. S1 in the supporting information). The regular pattern of squares was aligned with the cut primary flat of the wafer, which corresponds to the orientation of the crystal lattice planes, and mounted in soft contact mode (i.e. no direct contact between the mask and the resist on the wafer) using a mask aligner (MA6 Maskaligner, Karl Süss, Germany). The stack was exposed to UV light at a wavelength of $365 \mathrm{~nm}, 10 \mathrm{~mW}$ power. A multiple-exposure mode $(2 \times 10 \mathrm{~s}$ with a $15 \mathrm{~s}$ interval) was used to transfer the patterns from the specially designed polymeric photolithography mask (printed by Selba SA, Versoix, Switzerland) with a replication accuracy of $2 \mu \mathrm{m}$. Afterwards, the resist was developed in MF-321 solution (Microposit, USA) for $70 \mathrm{~s}$, rinsed with water and dried in a stream of nitrogen [Fig. 1(a), part (ii)]. In the next

(a)

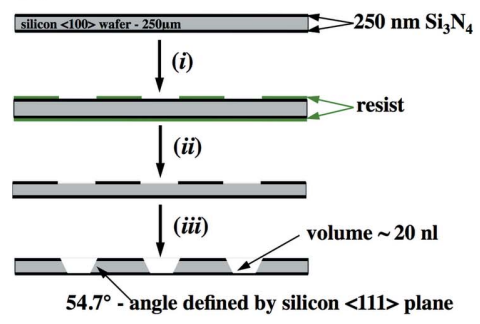

(b)

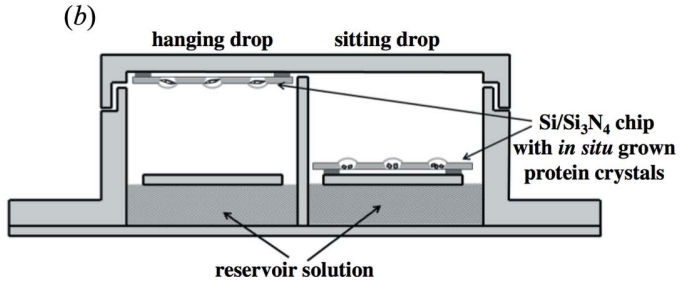

Figure 1

Microfabrication and use of a silicon chip with nanomembrane windows for serial crystallography. (a) The microfabrication steps carried out to obtain a multi-well array generated for in situ crystallization (MAGIC): (i) photolithography to transfer a geometric pattern to a resist layer; (ii) reactive ion etching to form $\mathrm{Si}_{3} \mathrm{~N}_{4}$ windows and resist removal in a solvent bath; (iii) potassium hydroxide etching to form thin $\mathrm{Si}_{3} \mathrm{~N}_{4}$ membranes. (b) Setup for crystallization experiments on $\mathrm{Si}_{3} \mathrm{~N}_{4}$ membrane chips in the hanging-drop (left) and sitting-drop (right) arrangements. (c) The asymmetric sandwich assembly employed to protect crystals during XFEL (or synchrotron beamline) experiments. Note that the applied geometry allows wide-angle data collection; chips with larger membrane windows are used as lids. step, $\mathrm{Si}_{3} \mathrm{~N}_{4}$ was completely removed from the areas not covered by the resist in a reactive-ion etching chamber (RIE 100, Oxford Instruments, UK) using fluoroform $\left(\mathrm{CHF}_{3}\right.$, gas flow rate 40 s.c.c.m.; s.c.c.m. = standard cubic centimetre per minute) and oxygen $\left(\mathrm{O}_{2}\right.$, gas flow rate 5 s.c.c.m. $)$ as the process gases. An inductively coupled plasma was applied at a forward power of $800 \mathrm{~W}$. Afterwards, the wafer was sequentially washed in filtered high-quality acetone (MOS PURANAL grade, Merck) and isopropanol (Micropur grade, Technic France) to remove the resist [Fig. 1(a), part (ii)]. Subsequently, anisotropic wet etching was performed in $20 \%$ $\mathrm{KOH}$ solution at $353 \mathrm{~K}$ for approximately $3.5 \mathrm{~h}$ to form freestanding $\mathrm{Si}_{3} \mathrm{~N}_{4}$ membranes [Fig. 1(a), part (iii)]. After this step, the wafer was immediately placed in water at $333 \mathrm{~K}$ and washed by soft agitation. All water used was of ultrapure quality (resistivity $18.2 \mathrm{M} \Omega \mathrm{cm}^{-1}$ ). The wafer was then washed in a fresh portion of water at $323 \mathrm{~K}$, followed by $5 \mathrm{~min}$ in $10 \%$ hydrochloric acid $(\mathrm{HCl})$ (diluted from $37 \% \mathrm{HCl}$, SigmaAldrich, Switzerland) at $313 \mathrm{~K}$ to avoid the formation of precipitates (contaminants) upon drying. Finally, the membranes were washed in water at room temperature and dried in a spin dryer (Polos SPIN150i/200i Infinite, SPSEurope BV, Netherlands) for $2 \mathrm{~min}$ at $500 \mathrm{r} \mathrm{min}^{-1}$, or using a nitrogen gun with the gas stream running parallel to the membranes. Single chips were separated from the full wafer to form individual substrates by cleaving along fine lines that were included in the initial pattern on the lithography mask and created by partial etching into the wafer that took place during the anisotropic wet etching step (Fig. 1a).

\subsection{Crystallization setup}

The vapour diffusion method was employed to grow protein crystals in situ on the $\mathrm{Si}_{3} \mathrm{~N}_{4}$ windows of the chips. Both sittingdrop (SD) and hanging-drop (HD) geometries were tested (Fig. 1b). Crystallization experiments were set up in doublebottomed transparent polymeric boxes (two-well $\mu$-Slide, ibidi, Germany). All prepared solutions were kept on ice before deposition. A $200 \mathrm{mg} \mathrm{ml}^{-1}$ stock solution of lysozyme from chicken egg white (AxonLab No. 9001-63-2, Switzerland) was prepared by gently agitating a suspension of the powder in water in a $1.5 \mathrm{ml}$ Eppendorf tube by means of a pipette. The stock was diluted further with water to give concentrations ranging from 25 to $100 \mathrm{mg} \mathrm{ml}^{-1}$.

A $20 \mu \mathrm{l}$ aliquot of the mixture of protein solution with precipitating agent (full details are given in Table S1 in the supporting information) was pipetted onto the fenestrated chip (a $14 \times 14$ window array placed with $1 \mathrm{~mm}$ pitch) with square membranes of $a=100,200,300,400$ or $500 \mu \mathrm{m}$ in size and spread over all 196 wells with a cut pipette tip held by curved tweezers. To enable monitoring of crystal growth without the risk of the sample drying out or overheating (melting) in the microscope light, chips with deposited solutions were mounted on the inner part of the ibidi box lid (HD geometry) or base (SD geometry) and secured with Scotch tape at the corners. 
A small spacer was used to ensure that the $\mathrm{Si}_{3} \mathrm{~N}_{4}$ membranes did not come into direct contact with the box. This was either a rolled piece of Scotch tape or a small piece of silicon wafer (thickness $250 \mu \mathrm{m}$ ) placed on a thin strip of the double-sided adhesive tape (thickness $100 \mu \mathrm{m}$ ) fixed to the inner side of the crystallization chamber lid or base. Several chemical conditions were tested, including the addition of a few microlitres of $50 \%$ polyethylene glycol (PEG, SigmaAldrich) in $\mathrm{H}_{2} \mathrm{O}$ or $100 \%$ ethylene glycol (EG, SigmaAldrich) to the aqueous lysozyme solution (full details are given in Table $\mathrm{S} 1$ in the supporting information). A $1 \mathrm{ml}$ aliquot of reservoir solution (precipitating agent) prepared from $1 M$ sodium acetate (NaAc, Sigma-Aldrich) pH 4.5, $3 \mathrm{M}$ sodium chloride $(\mathrm{NaCl}$, Sigma-Aldrich) and water in the proportion 1:2:7, resulting in final concentrations of $0.1 \mathrm{M}$ $\mathrm{NaAc}$ and $0.6 \mathrm{M} \mathrm{NaCl}$, was pipetted at the very bottom of the ibidi boxes (Fig. 1b). After incubation for several hours to allow crystal growth, the chips were imaged with a Leica M205C stereo microscope (Mannheim, Germany).

\subsection{Automatic deposition of the crystallization solutions}

To have more control over the sample volume, a dispensing device equipped with a microcapillary nozzle and able to handle liquid volumes down to $5 \mathrm{nl}$ (Arnold et al., 2016) was used to deposit the sample in the individual wells of a silicon chip. To avoid evaporation of minute amounts of the deposited solutions, the silicon chip was placed on a temperaturecontrolled stage and cooled to the dew point of the environment during this process. Initially, $500 \mathrm{nl}$ of the solution to be dispensed were aspirated by a dispensing nozzle (New Objective, USA, FS360-100-30, inner diameter $100 \mu \mathrm{m}$ with a tapered tip of $30 \mu \mathrm{m}$ diameter) with a flow rate of $1 \mu \mathrm{min}^{-1}$. The wells, with volumes of about $20 \mathrm{nl}$, were filled either with 50:50 mixtures of an aqueous $50 \mathrm{mg} \mathrm{ml}^{-1}$ lysozyme solution and precipitating agent solution with the same composition as the reservoir solution above $(0.1 M \mathrm{NaAc}, 0.6 M \mathrm{NaCl}, \mathrm{pH}$ 4.5 ) or in sequential order (first with the protein and then with the precipitating agent solution or vice versa) with a deposition flow rate of $10 \mu \mathrm{min}^{-1}$. Once loaded, the chips were mounted in a large VDX 24-well crystallization plate (Hampton Research) in the SD geometry and incubated at room temperature versus $0.5 \mathrm{ml}$ of reservoir solution placed at the bottom of the plate wells. After several hours of incubation at room temperature, they were observed using a light microscope (Axiophot, Zeiss, USA) to assess crystal growth.

\subsection{Screening crystallization conditions on the chip}

To demonstrate the feasibility of using the $\mathrm{Si}_{3} \mathrm{~N}_{4}$ membrane chips for screening experiments carried out to establish the optimal conditions for crystal growth, a chip with $500 \times$ $500 \mu \mathrm{m}$ membranes was loaded with the following lysozyme solutions: (i) lysozyme at a concentration of $50 \mathrm{mg} \mathrm{ml}^{-1}$ in $50 \mathrm{~m} M \mathrm{NaAc}$ buffer $\mathrm{pH} 4.5$; (ii) the solution in (i) plus $50 \%$ PEG aqueous solution and reservoir solution (0.1 $M \mathrm{NaAc}$ and $0.6 M \mathrm{NaCl} \mathrm{pH} 4.5$ ) in the proportions 9:1:1; or (iii) lysozyme at a concentration of $50 \mathrm{mg} \mathrm{ml}^{-1}$ in $\mathrm{H}_{2} \mathrm{O}$. A pipette was used to deposit $8 \mu \mathrm{l}$ aliquots of the solutions on the different regions of the chip. Each single drop covered approximately 25 wells. Once loaded, the chips were mounted in HD geometry in an ibidi box as described above, incubated versus $1 \mathrm{ml}$ of reservoir solution and imaged after $8 \mathrm{~h}$ using a Leica ML95 light microscope (Mannheim, Germany) equipped with a Moticam 5.0 MP camera (Motic, Hong Kong).

\subsection{Sandwich assembly}

Double-sided $100 \mu \mathrm{m}$ thick adhesion tape (Laminex spacer, Molecular Dimensions, MD11-50) was used to assemble asymmetric (contact well to membrane side) watertight chambers out of two chips. A thin adhesive frame ( $2 \mathrm{~mm}$ wide) was cut out of a continuous foil and placed on one side of one chip. This chip serving as a lid was first fixed, adhesive side up, on a cube of soft Styrofoam-like material (Hobbyatelier Brugg, Switzerland) using $400 \mu \mathrm{m}$ thick needles inserted through $400 \times 400 \mu \mathrm{m}$ square orifices ('knockout' membranes; see Fig. S1 in the supporting information) at its corners. A second grid was used for an SD or HD crystallization run. Once the run was complete, the second grid with crystals grown on its membrane was aligned to the lid using the needles and squeezed gently together around the frame with tweezers to form a sealed 'sandwich' (Fig. 1c).

\subsection{Structure refinement from the synchrotron diffraction data collected on in situ grown crystals}

Diffraction data from several lysozyme crystals prepared in HD geometry (see Table S1 in the supporting information, chip 48) and enclosed in a sandwich were collected at room temperature on the X06SA PX-I beamline at the Swiss Light Source at the Paul Scherrer Institute, Switzerland, using a $40 \times$ $20 \mu \mathrm{m}$ beam at a wavelength of $1.00 \AA$. The full flux of $1.5 \times$ $10^{12}$ photons s ${ }^{-1}$ was attenuated to $0.3 \%$ for data collection. Data were collected with an EIGER X 16M photon-counting detector placed $200 \mathrm{~mm}$ from the sample with a rotation of $0.1^{\circ}$ per frame and exposure of $0.1 \mathrm{~s}$ per frame. Owing to geometric constraints, the total rotation range of the chip was limited to about $50^{\circ}$. Data from two individual crystals from one chip were merged to obtain a full data set. Data indexing was done with the program package $X D S$ (Kabsch, 2010b), and scaling and merging with XSCALE (Kabsch, 2010a). Structure solution was done by molecular replacement with the program MOLREP (Vagin \& Teplyakov, 1997), using the Protein Data Bank (PDB) code 2lyz as search model. Structure refinement was done using REFMAC (Murshudov et al., 1997), BUSTER (Bricogne et al., 2016) and phenix.refine (Afonine et al., 2012), and model validation using MOLPROBITY (Chen et al., 2010).

\section{Results and discussion}

\subsection{Chip design}

The chip layout (Fig. S1 in the supporting information) was designed for serial femtosecond crystallography, which requires several hundreds to thousands of similarly placed 
crystals. A number of quality and microfabrication requirements influenced the final geometry of the chip. The chip had to (i) be compatible with double-bottomed crystallization chambers (Fig. 1c), (ii) be strong enough to be handled manually during crystallization experiments and mounting in the XFEL beam, (iii) facilitate in situ crystallization, (iv) allow the microwells to be sealed by stacking two chips, and (v) enable fast shifts from window to window during data acquisition. Regarding these points, we found the following limitations: the geometry of the wells is defined by the anisotropic etching process and the angle between the crystallographic planes of the single-crystal silicon; in addition, the applied photolithographic process requires the application of sufficiently wide lines (at least $50 \mu \mathrm{m}$ ) between the patterned features, limiting the well density. For example, a minimal distance of $550 \mu \mathrm{m}$ is required between the centres of $200 \mu \mathrm{m}^{2}$ windows in a $250 \mu \mathrm{m}$ thick silicon wafer. Thinner wafers are much more difficult to handle during the microfabrication processes, as silicon is brittle and fragile.

The overall size of the chips was $17 \times 17 \mathrm{~mm}$, so that they would fit into $20 \times 20 \mathrm{~mm}$ crystallization chambers (Fig. 1 b). They were patterned with fully symmetric arrays of 196 square-bottomed cavities $(14 \times 14)$ placed with a distance of $1 \mathrm{~mm}$ between the centres of the squares (Fig. S1 in the supporting information). The microfabrication process started with polished silicon $\langle 100\rangle$ wafers coated with a $250 \mathrm{~nm}$ thick layer of $\mathrm{Si}_{3} \mathrm{~N}_{4}$ on both sides, and consisted of three major steps: (i) photolithography on the reverse of the wafer to define the pattern; (ii) reactive ion etching to transfer the pattern into the $\mathrm{Si}_{3} \mathrm{~N}_{4}$; and (iii) anisotropic wet etching in $20 \%$ $\mathrm{KOH}$ solution through the silicon wafer to create deep cavities defined by the thickness $(250 \mu \mathrm{m})$ of the silicon wafer (Fig. $1 a$, and see §2.1). Their mechanically stable bottoms were formed by the remaining 30-250 $\mathrm{nm}$ thick amorphous low-stress and smooth $\mathrm{Si}_{3} \mathrm{~N}_{4}$ layer. The square membranes had an area of $40000-250000 \mu \mathrm{m}^{2}$ and were uniform in size over each chip. The wells had an approximate volume of 38-117 $\mathrm{nl}$, which gives an overall chip capacity between 7448 and $22932 \mathrm{nl}$ when the wells are filled to the rim. Chips with larger (500 and $1000 \mu \mathrm{m}) \mathrm{Si}_{3} \mathrm{~N}_{4}$ membrane windows could be used as 'lids', i.e. they were stacked on top and sealed (Fig. 1c), allowing wideangle data collection. Large $\mathrm{Si}_{3} \mathrm{~N}_{4}$ membranes, which formed at defined positions in all four corners of the $2 \mathrm{~mm}$ wide outer frame of every chip at an equal distance from the edge (Fig. S1 in the supporting information), were removed with a thin needle. These openings served as alignment aids when asymmetric sandwiches were assembled (Fig. 1c).

\subsection{In situ crystallization}

The chips described above were used as solid supports for the in situ crystallization of lysozyme and fixed-target XFEL data collection. Direct crystallization on ultrathin $\mathrm{Si}_{3} \mathrm{~N}_{4}$ freestanding films by vapour diffusion was performed in the HD and SD geometries (Fig. 1b). The first crystals were usually visible under a light microscope a few hours after deposition of the crystallization solution (minimum $6 \mathrm{~h}$ ).
The size and crystal form of the growing crystals depended on the crystallization conditions in the silicon wells. Crystals with different shapes and sizes were obtained and were positioned in a wide range of crystallographic orientations on the $\mathrm{Si}_{3} \mathrm{~N}_{4}$ membranes; full details are given in Table $\mathrm{S} 1$ in the supporting information. The average coverage of the wells with crystals of the desired size was more than $90 \%$.

The balance between the formation of new crystals and crystal growth is related to the amount of protein available. In the vapour diffusion method, the protein and salt concentrations increase as the drop equilibrates with the reservoir solution. Once the protein reaches supersaturation, nucleation and crystal growth are in competition. It is known that several critical factors affect the dimensions of the protein crystals obtained (Falkner et al., 2005). The concentration of a protein in the crystallization solution is an important variable. When the concentration was too low in the HD and SD experiments, the nucleation rate was low and few crystals grew. This low crystal density led to insufficient coverage of the membrane, but usually favoured the formation of large crystals. When the protein concentration was too high, nucleation rates were high, leading to clusters of many small crystals. When the concentration of the protein was much too high, i.e. close to the solubility limit, precipitation without crystal formation was observed.

During crystallization experiments in the chamber, the difference between the concentration in the protein solution and that in the reservoir solution was the driving force that caused water to evaporate from the drop. This led to nucleation and further crystal growth. The crystallization process proved to be critical for the mechanical stability of freestanding ultrathin $\mathrm{Si}_{3} \mathrm{~N}_{4}$ membranes during the incubation period (Fig. S2 in the supporting information), which also depended on their thickness, uniformity and lack of pinholes, i.e. the quality of the CVD coating. The very thin intact membranes $(30 \mathrm{~nm})$ fabricated initially tended to break. The probability of nucleation and the growth of crystals were increased in the corners of the wells, as smaller crystals were observed to cluster in these areas. Presumably, the membranes broke when the forces exerted on the film were too high during formation of the crystals. As a result, samples intended for time-resolved XFEL measurements were prepared on $250 \mathrm{~nm}$ thick membranes.

\subsection{Multiwell silicon chips for screening crystallization conditions}

Microfabricated multiwell chips are suitable for nanovolume crystallization screening and allow the optimum crystallization conditions to be determined with minimal protein consumption. Their use together with XFELs promises to facilitate the structural analysis of proteins that are difficult to obtain in large quantities. As an initial proof of concept, $8 \mu \mathrm{l}$ portions of three lysozyme solutions in buffers with different $\mathrm{pHs}$ were pipetted by hand onto selected areas of a multiwell chip. Each sample covered an area of approximately 25 wells. Crystallization was carried out in the 


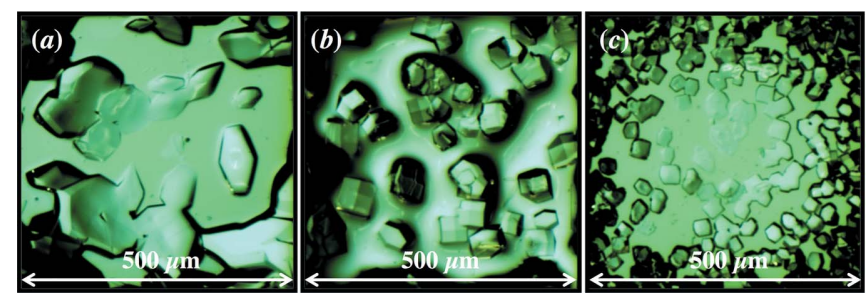

Figure 2

Crystals grown under different conditions on $\mathrm{Si}_{3} \mathrm{~N}_{4}$ membrane windows of the same chip. The size and shape of the crystals obtained depended on the composition of the sample buffer. (a) Lysozyme concentration $50 \mathrm{mg} \mathrm{ml}^{-1}$ in $50 \mathrm{mM} \mathrm{NaAc}$ buffer $\mathrm{pH}$ 4.5. (b) Solution as in part (a) modified with $50 \%$ PEG solution and reservoir solution $(0.1 M$ NaAc and $0.6 \mathrm{M} \mathrm{NaCl} \mathrm{pH} \mathrm{4.5)} \mathrm{in} \mathrm{the} \mathrm{proportion} \mathrm{9:1:1.} \mathrm{(c)} 50 \mathrm{mg} \mathrm{ml}^{-1}$ lysozyme solution in MilliQ $\mathrm{H}_{2} \mathrm{O}$.

HD geometry. Crystals were evident after $8 \mathrm{~h}$, and had different morphologies (habits) in the three regions of the chip (Fig. 2).

\subsection{Crystals from automated nanolitre dispension}

Nanolitre sample volumes have to be dispensed to make full use of the chips for screening and to allow the crystallization conditions to be varied from well to well. This requires the use of a robot such as the one described by Arnold et al. (2016). This automated unit was employed in the present study and is equipped with a glass capillary nozzle with a $30 \mu \mathrm{m}$ orifice (inner diameter), and with a dew-point stage to reduce liquid evaporation (Fig. 3). It was used to deposit precise volumes ranging from 5 to $15 \mathrm{nl}$ into each well. As the capacity of the wells used in these experiments was approximately $22 \mathrm{nl}$, the concave meniscus formed by the liquid was below the rim and mixing of the solutions deposited in different wells was avoided.

The deposition of nanolitre-volume drops from the tip of the nozzle proved difficult, especially when dispensing $5 \mathrm{nl}$ volumes. The droplet crept along the nozzle and foreclosed deposition in the silicon well. It has been reported that the application of a hydrophobic coating to the nozzle can be used to overcome this problem (Arnold et al., 2016). Alternatively,
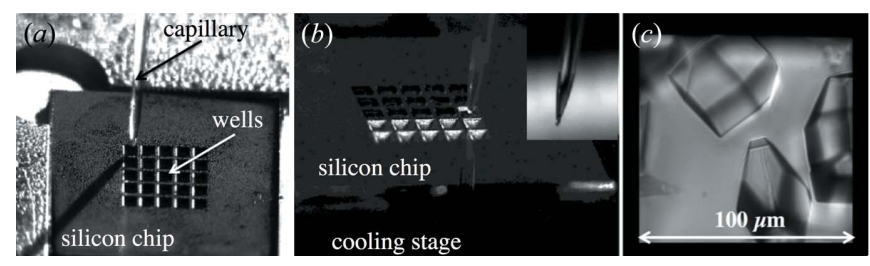

Figure 3

Crystal growth in nanolitre wells. (a) Top view of the silicon chip on the dew-point stage. The $5 \times 5$ well array is $2 \times 2 \mathrm{~mm}$ in size. $(b)$ Nanolitre wells being filled with protein solution by contact-mode deposition. The dark wells at the top are filled, while the bright wells at the bottom are empty. It took about 2 min to fill the array. The inset shows the capillary when moved away from the chip surface. (c) Lysozyme crystals grown on the $100 \times 100 \mu \mathrm{m} 30 \mathrm{~nm}$ thin $\mathrm{Si}_{3} \mathrm{~N}_{4}$ membranes from deposited nanolitre volumes of $50 \mathrm{mg} \mathrm{ml}^{-1}$ lysozyme mixed 1:1 with precipitating agent (0.1 $M \mathrm{NaAc}, 0.6 \mathrm{M} \mathrm{NaCl}, \mathrm{pH} 4.5)$. Crystallization was carried out in the SD geometry. contact-mode deposition of $15 \mathrm{nl}$ volumes was employed. This was achieved by touching the inclined wall of the silicon well, formed by anisotropic etching of the single-crystal chip, with the tip of the nozzle and allowing the liquid to flow down the sides to the $\mathrm{Si}_{3} \mathrm{~N}_{4}$ membrane at the bottom (Fig. 3b) (a short film showing the deposition is provided as supporting information). Contact between the nozzle and the fragile membrane was avoided. However, this approach increased the time required to fill the chip.

\subsection{Crystal growth optimization for time-resolved measure-} ments at the FEL

Protein crystals grown in situ as described in \$3.2, and protected from dehydration by a second $\mathrm{Si}_{3} \mathrm{~N}_{4}$ membrane as described in $\$ 2.5$, were used to investigate radiation damage processes in the femtosecond range at the FEL X-ray source at the Linac Coherent Light Source (LCLS, Menlo Park, California, USA; \$3.6). In the course of the crystallization trials (Table S1 in the supporting information), crystal growth was optimized to maximize the probability of X-ray interaction with the crystals, i.e. to maximize the expected hit rate during data collection. Conditions giving the maximum coverage of the membrane window by crystals with large lateral dimensions $(150-200 \mu \mathrm{m})($ Fig. $4 a)$ or by a high density of smaller crystals (approximately $50 \mu \mathrm{m}$ ) (Fig. 4b) were sought. The crystals observed on $200 \times 200 \mu \mathrm{m}$ membranes were usually the largest and had the optimum dimensions and distribution density for time-resolved measurements (Fig. 4a).

A strong influence of the crystallization solution storage temperature before deposition on the chip on the crystal growth was observed. Performing crystallization at room temperature from solutions previously stored at $277 \mathrm{~K}$

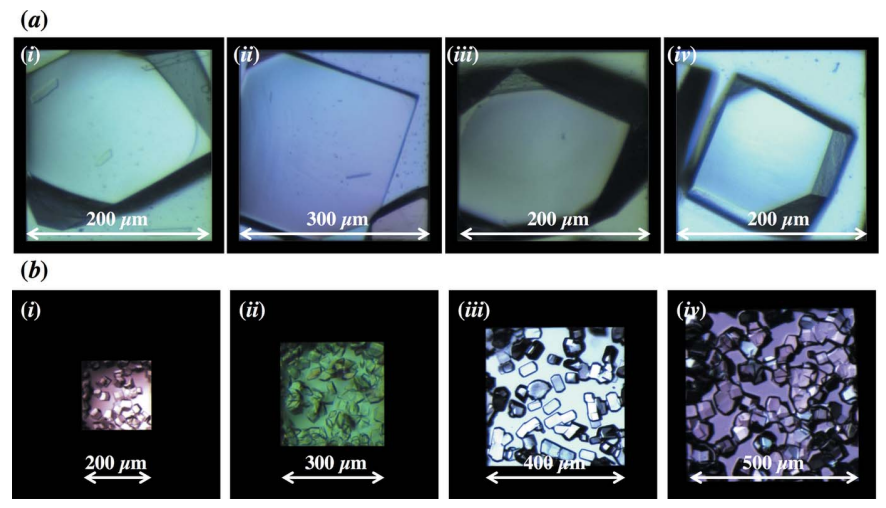

Figure 4

In situ crystal growth on $\mathrm{Si}_{3} \mathrm{~N}_{4}$ membranes with density optimization. (a) Single protein crystals grown on membranes filling the majority of the membrane window, the ideal case that will provide an almost $100 \%$ hit rate with serial diffraction data collection. The crystals shown were grown (HD geometry) at room temperature under different conditions, as detailed in Table S1 in the supporting information: (i) chip 30, (ii) chip 31, (iii) chip 20, (iv) chip 36. (b) Protein crystals grown (HD geometry) at room temperature from solutions (mixtures with precipitating agent) incubated beforehand overnight at $277 \mathrm{~K}$ are shown on membranes of various sizes. The conditions are detailed in Table S1 in the supporting information: (i) chip 11, (ii) chip 12, (iii) chip 4, (iv) chip 6. 
overnight usually resulted in growth of a higher density of smaller crystals compared with experiments run under the same conditions with freshly prepared solutions (Fig. S3 in the supporting information). It turned out that storage at lower temperatures leads to protein aggregation and the formation of clusters of protein acting as nucleation centres as soon as supersaturation is reached. These clusters seem to be stable enough to sustain an elevated temperature during setup of the crystallization. Increased density and reduced size of crystals grown from solutions conditioned at lower temperatures were observed independent of other parameters, including the size of the membrane (Fig. $4 b$ ).

Growth initiated from the window corners was often observed. As a consequence, using much larger membrane windows with single crystals resulted in poor coverage of the centre of the window (Fig. S5 in the supporting information). Therefore, when aiming for high coverage of the membrane by single crystals, the crystal size and window should be similar. Growth initiating from the window corners can also lead to maximal coverage of the membrane, when a single crystal grows over the single membrane and the crystal size matches the membrane dimensions.

As expected, the chemical composition of the salt solution was a further important crystallization parameter. Using salt solutions of different $\mathrm{pH}$ is known to influence the crystallization of lysozyme, leading to different morphologies (Basso et al., 2005). The final size of the crystals obtained is also affected by the $\mathrm{pH}$ value, since it is directly related to the net surface charge of the molecule (isoelectric point). Moreover, additives such as PEG or EG that interact with the solvation shell of protein molecules have an impact on the final crystal shape. Indeed, the addition of such surface-active substances in the present study modified the shape of the protein crystals formed and tended to change their aspect ratio, resulting in elongated rod-like crystals (Fig. 5).

\subsection{Assessment of in situ grown crystal quality by synchrotron radiation}

In order to assess the quality of lysozyme crystals grown in situ and their preservation in the sandwich-type enclosure (§2.5), diffraction experiments were carried out at room

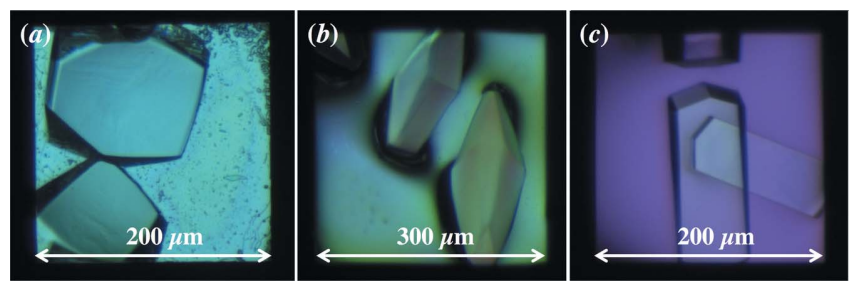

Figure 5

The influence of additives on the direct crystallization of lysozyme on $\mathrm{Si}_{3} \mathrm{~N}_{4}$ membranes. The presence of additives in the protein buffer changed the aspect ratio of the crystals. (a) $50 \mathrm{mg} \mathrm{ml}^{-1}$ lysozyme in $50 \mathrm{~m} M$ NaAc buffer. (b) As in part (a) but plus PEG. (c) As in part (a) but plus EG. For details, see Table S1 in the supporting information, chips (a) 20, (b) 17 and (c) 13 .

Table 1

Data collection (beamline PX-I) and refinement statistics for in situ grown lysozyme crystals.

Data collection

Temperature (K)

Wavelength (犬), phasing method

Total range collected per crystal $\left(^{\circ}\right)$

Number of crystals merged

Space group

Unit-cell parameters $a, b, c(\AA)$, $\alpha=\beta=\gamma\left({ }^{\circ}\right)$

Resolution ( $\AA$ )

$R_{\text {meas }}$

$\langle I / \sigma(I)\rangle$

Completeness

$\mathrm{CC}_{1 / 2}$

Mosaicity $\left({ }^{\circ}\right)$

\section{Refinement}

$R_{\text {work }} / R_{\text {free }}$

Bond lengths r.m.s. $(\AA)$, bond angles r.m.s. $\left(^{\circ}\right)$

Ramachandran favoured (\%)

Ramachandran outliers (\%)

Rotamer outliers (\%)

Clash score

Average $B$ factor $\left(\AA^{2}\right)$

PBD code
293

1.00 , molecular replacement 50

2

$P 4_{3} 2{ }_{1} 2$

79.50, 79.50, 37.81, 90

50-1.57 (1.61-1.57)

$0.05(0.847)$

$13.74(1.04)$

$0.954(0.952)$

$99.9(70.5)$

0.033 temperature on the PX-I beamline at the Swiss Light Source (PSI). Data were collected from individual 50-100 $\mu \mathrm{m}$ crystals selected using an inline microscope (Fig. S6 in the supporting information) on a chip assembly that was mounted on the goniometer head. The sample could be tilted by about $\pm 30^{\circ}$ without shadowing the crystals by the supports. Data were collected under crystal rotation by about $50^{\circ}$, leading to highquality data sets (Table 1), although with limited completeness depending on the crystal orientation.

Merging data sets from two tetragonal crystals with different orientations on the support yielded complete crystallographic data at $1.57 \AA$ resolution. Structure solution and refinement provided a high-quality electron-density map (Fig. 6). For full data set statistics and refinement details, see
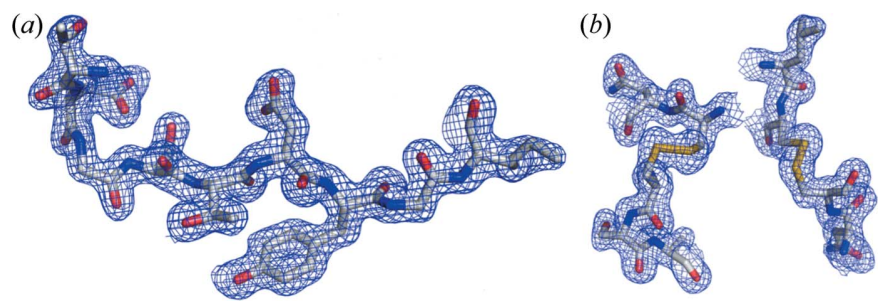

Figure 6

Details of the electron density obtained from the $1.57 \AA$ roomtemperature data set collected from lysozyme crystals enclosed between silicon nitride membranes (PDB code 5ne0). (a) Residues THR47ILE55. (b) Disulfide bonds between CYS64-CYS80 and CYS76-CYS94. The difference maps showed no residual density on the disulfide bonds, which indicates that the bonds remained intact and that the protein structure suffered no significant radiation damage. The $2 F_{\mathrm{o}}-F_{\mathrm{c}}$ maps are shown as blue meshes contoured at $1 \sigma$. Stick models show $\mathrm{N}$ atoms (blue), $\mathrm{O}$ atoms (red), $\mathrm{S}$ atoms (yellow) and $\mathrm{C}$ atoms (grey). 
Fig. S7 and Table S2 in the supporting information. All quality parameters of the obtained data and model compare favourably with other lysozyme entries in the PDB. The low mosaicity given by $X D S\left(0.033^{\circ}\right)$ and the sharpness of the peaks (Fig. S7 in the supporting information) are consistent with room-temperature crystals in a good state of conservation. This proves that the crystals were satisfactorily protected by the silicon nitride enclosure.

\subsection{Sample preparation for and the collection of time- resolved diffraction data}

Large protein crystals on solid supports were produced for time-resolved measurements aimed at the investigation of $\mathrm{X}$-ray induced damage in the femtosecond range using a splitdelay X-ray optical setup similar to the one described earlier (David et al., 2015). A detailed report, including a full description of the experiment and the results of the ongoing data evaluation, is in preparation. In brief, fractions of an X-ray pulse delivered by the XFEL were split off using a set of diamond gratings in the optical path. A second set of gratings redirected the split beams to the sample, where they arrived under an angle of up to $6 \mathrm{mrad}$ and with a time delay in the range of 20-300 fs. The setup was designed in such a way that one set of split beams overlapped with the 'direct' primary pulse on the sample (pump-and-probe area), while a second symmetric set of split beams was focused on an area approximately $70 \mu \mathrm{m}$ away (reference area). The setup was adapted to diffraction geometry where the primary 'pumping' beam and the probing and reference beams were diffracted by the same Bragg plane within the protein crystal. The slightly
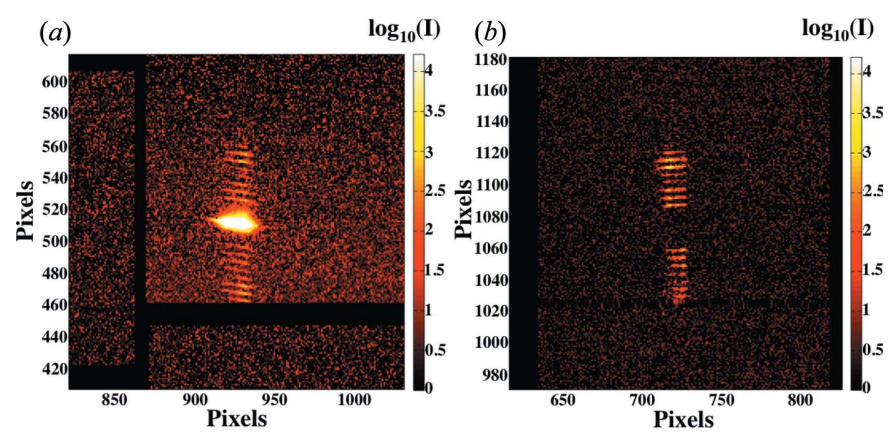

Figure 7

Examples of diffraction data collected on a CSPAD pixellated detector (Hart et al., 2012) during time-resolved pump-probe experiments with the split-delay X-ray optics line on large lysozyme crystals. (a) The set of Bragg reflections stemming from the pump beam (central intense peak) and the decreased intensity delayed peaks. $(b)$ The pumping beam has been blanked out, leading to sets of delayed peaks in both the probing (top) and the reference (bottom) region. The views shown here are limited to a small part of the detector but they contain the full signal registered during single shots (the detector was placed off-axis in the horizontal direction at an angle of approximately $13^{\circ}$ from the direct beam and at a distance of $122 \mathrm{~cm}$ from the sample). Images are plotted on a $\log _{10}$ scale and pixels with intensity $I<1$ counts were set to 0 . A high contrast was obtained between the registered signal and the background owing to the high X-ray transmission of the used $\mathrm{Si}_{3} \mathrm{~N}_{4}$ membranes. The membrane supporting the crystals and the membrane in the sandwich lid were each $250 \mathrm{~nm}$ thick. different incident angles of the delayed pulses translate into slightly different positions on the detector (delayed peaks). Optimization of the sample-to-detector distance for the resolution of the delayed peaks led to limitation of the accessible $q$ range (8-14.5 $\AA$ ). As a consequence, only single Bragg peaks (with their accompanying delayed peaks) were observed from individual X-ray pulses.

The samples were kept at room temperature and atmospheric pressure. Being sealed between two $\mathrm{Si}_{3} \mathrm{~N}_{4}$ windows (Fig. 1c, \$2.5) prevented them from dehydrating. The chip 'sandwich' was mounted on a fast $X Y$ stage, aligned, and moved stepwise through the XFEL beam (step size $1 \mathrm{~mm}$ ) to irradiate the membrane array window by window (\$3.1) at a rate of up to $1 \mathrm{~Hz}$ until the whole sample area had been covered $(14 \times 14 \mathrm{~mm})$. Under these conditions, Bragg reflections and the time-delayed peaks were collected with sufficient spatial resolution and with very good contrast owing to the high X-ray transparency of the $\mathrm{Si}_{3} \mathrm{~N}_{4}$ membranes (Fig. 7a). The reference experiments with blanked primary beams showed only the delayed peaks (Fig. $7 b$ ). The high quality of the registered signal showed that the protection provided by the 'sandwich' chip assembly was sufficient to prevent dehydration of the crystals before the measurement. Furthermore, the single crystals were large enough to be hit simultaneously by the two sets of beams separated by $70 \mu \mathrm{m}$. However, the samples dried out slowly during data collection, because the pulses of intense X-rays created holes in the $\mathrm{Si}_{3} \mathrm{~N}_{4}$ membranes leading to slow evaporation from adjacent wells. Sealing each of the wells individually would help to overcome this problem. Application of the other materials commonly used for in situ data collection at synchrotrons (like e.g. cyclic olefin copolymer or Kapton foil, see Fig. S8 in the supporting information) would significantly increase the background, especially given their partially ordered structures, and would most likely indispose the observation of weak delayed peaks. Moreover, very thin plastic foils are water permeable and not suitable for long-term data collection. Also, the previously reported examples of fixed targets, i.e. vapour diffusion crystallization plates or microfluidic chips [as described by Feld et al. (2015), Huang et al. (2015, 2016), Axford et al. (2016), Lyubimov et al. (2015) and Murray et al. (2015)] provide much higher background levels, mainly due to the applied thicknesses and/or (semi-)crystalline materials. Silicon nitride fabrication technology is unique in this respect in providing thin films in the sub-micrometre range, which gives an ultralow continuous (amorphous) background in X-ray diffraction, as shown in Fig. S8 in the supporting information.

\section{Conclusions and outlook}

Protein crystals of the desired size could be efficiently grown with the required distribution density on $\mathrm{Si}_{3} \mathrm{~N}_{4}$ membrane substrates, protected from dehydration by the formation of an $\mathrm{Si}_{3} \mathrm{~N}_{4}$ membrane chamber and analysed in situ by an XFEL beam. This opens many possibilities for the time-resolved investigation of phenomena occurring in the femtosecond regime. The $\mathrm{Si}_{3} \mathrm{~N}_{4}$ membranes employed are highly X-ray 
transparent (composed of light chemical elements), ultrathin, self-supporting, stable and water impermeable, and allow the production of multiple copies of large protein crystals at defined places for serial measurement in quasi-random crystallographic orientations. In an ideal case, the chips would provide a $100 \%$ guarantee of hitting the crystalline sample during data collection at an XFEL, i.e. when the membrane windows are completely covered by single crystals grown with lateral dimensions matching the window size or with a high density of small crystals.

The applied technology has the potential for the production of supports with about one order of magnitude higher density of the wells using thinner wafers and adapted designs. This makes them attractive for application at XFELs with repetition rates in the $100 \mathrm{~Hz}$ range, such as at the planned fixedtarget instrument for protein crystallography at Experimental Station B of the SwissFEL. Using a few tens of chips of area $1 \times 1 \mathrm{~cm}$ should be sufficient for the collection of a full data set of an unknown protein. However, when addressing higher repetition rate sources an even higher sample density is required, which could for instance be achieved with elongated windows (Hunter et al., 2014) in which a whole series of crystals are sequentially probed during a fast one-directional movement.

Given the notable improvements that allow the more efficient collection of diffraction data from fixed targets at XFEL sources (Feld et al., 2015), the key to obtaining high-resolution protein structures now lies in the material quality and the sample preparation and handling procedures. When crystals are grown on the fixed-target support they can be delivered directly to the beam. In situ measurements avoid the mechanical stress incurred during handling and thus help to maintain the quality of the sample until data collection.

Importantly, the method used to grow crystals on $\mathrm{Si}_{3} \mathrm{~N}_{4}$ membranes significantly reduces the sample volume required. In particular, automatic handling of the liquid allows for nanolitre deposition and for crystallization conditions to be screened on the nanolitre scale.

The principles and techniques presented here can be applied to a wide range of proteins and promise to extend the list of structures solved using XFELs. In the future, this crystallization procedure, which requires minimal volumes, could be adapted for nanocrystal growth as well as for deposition of crystal suspensions. In spite of the demonstrated success of the method described here, the use of polymers and other materials to make the chips easier to mass-produce and to reduce their cost needs to be investigated further (Feld $e t$ al., 2015). The membranes should be composed of light elements so that they are essentially transparent to the X-ray beam, thin to reduce absorption and diffuse scattering, and less brittle than $\mathrm{Si}_{3} \mathrm{~N}_{4}$ membranes.

\section{Accession code}

The coordinates and structure factors of the roomtemperature structure of lysozyme between silicon nitride membranes have been deposited in the PDB under accession code 5 ne 0 .

\section{Acknowledgements}

The authors would like to thank Dario Marty and Christopher Wild for technical assistance in microfabrication. We are grateful to Marcin Sikorski and his team for experiment support and data acquisition at the XCS endstation. Parts of this research were carried out at the Linac Coherent Light Source (LCLS) at the SLAC National Accelerator Laboratory. LCLS is an Office of Science User Facility operated for the US Department of Energy Office of Science by Stanford University. Use of the LCLS, SLAC National Accelerator Laboratory, is supported by the US Department of Energy, Office of Science, Office of Basic Energy Sciences, under contract No. DE-AC02-76SF00515. We also acknowledge the Paul Scherrer Institut, Switzerland, for provision of synchrotron radiation beamtime on beamline PX I of the SLS. Istvan Mohacsi and Shirley Müller helped in manuscript preparation.

\section{Funding information}

Funding for this research was provided by: Swiss Nanoscience Institute (project No. P1305).

\section{References}

Afonine, P. V., Grosse-Kunstleve, R. W., Echols, N., Headd, J. J., Moriarty, N. W., Mustyakimov, M., Terwilliger, T. C., Urzhumtsev, A., Zwart, P. H. \& Adams, P. D. (2012). Acta Cryst. D68, 352-367.

Arnold, S. A., Albiez, S., Opara, N., Chami, M., Schmidli, C., Bieri, A., Padeste, C., Stahlberg, H. \& Braun, T. (2016). ACS Nano, 10, 49814988.

Auer, M., Scarborough, G. A. \& Kühlbrandt, W. (1999). J. Mol. Biol. 287, 961-968.

Axford, D., Aller, P., Sanchez-Weatherby, J. \& Sandy, J. (2016). Acta Cryst. F72, 313-319.

Basso, S., Fitch, A. N., Fox, G. C., Margiolaki, I. \& Wright, J. P. (2005). Acta Cryst. D61, 1612-1625.

Baxter, E. L. et al. (2016). Acta Cryst. D72, 2-11.

Billeter, M., Wagner, G. \& Wüthrich, K. (2008). J. Biomol. NMR, 42, 155-158.

Binshtein, E. \& Ohi, M. D. (2015). Biochemistry, 54, 3133-3141.

Bricogne, G., Blanc, E., Brandl, M., Flensburg, C., Keller, P., Paciorek, P., Roversi, P., Sharff, A., Smart, O., Vonrhein, C., Womack, T. (2016). BUSTER. Version 2.10.2. Global Phasing Ltd, Cambridge, England.

Chapman, H. N. et al. (2007). Nature, 448, 676-679.

Chen, V. B., Arendall, W. B. III, Headd, J. J., Keedy, D. A., Immormino, R. M., Kapral, G. J., Murray, L. W., Richardson, J. S. \& Richardson, D. C. (2010). Acta Cryst. D66, 12-21.

Cohen, A. E. et al. (2014). Proc. Natl Acad. Sci. USA, 111, 1712217127.

Conrad, C. E. et al. (2015). IUCrJ, 2, 421-430.

Coquelle, N., Brewster, A. S., Kapp, U., Shilova, A., Weinhausen, B., Burghammer, M. \& Colletier, J.-P. (2015). Acta Cryst. D71, 11841196.

David, C., Karvinen, P., Sikorski, M., Song, S., Vartiainen, I., Milne, C. J., Mozzanica, A., Kayser, Y., Diaz, A., Mohacsi, I., Carini, G. A., Herrmann, S., Färm, E., Ritala, M., Fritz, D. M. \& Robert, A. (2015). Sci. Rep. 5, 7644.

Falkner, J. C., Al-Somali, A. M., Jamison, J. A., Zhang, J., Adrianse, S. L., Simpson, R. L., Calabretta, M. K., Radding, W., Phillips, G. N. Jr \& Colvin, V. L. (2005). Chem. Mater. 17, 2679-2686. 
Feld, G. K. et al. (2015). J. Appl. Cryst. 48, 1072-1079.

Frank, M. et al. (2014). IUCrJ, 1, 95-100.

Fromme, P. (2015). Nat. Chem. Biol. 11, 895-899.

Gibb, T. \& Ayub, M. (2013). Solid-State Nanopore Fabrication. Engineered Nanopores for Bioanalytical Applications, edited by J. B. Edel \& T. Albrecht, ch. 5, pp. 121-140. Amsterdam: Elsevier. Hart, P. et al. (2012). IEEE NSS/MIC, 2012, 538-541.

Huang, C.-Y., Olieric, V., Ma, P., Howe, N., Vogeley, L., Liu, X., Warshamanage, R., Weinert, T., Panepucci, E., Kobilka, B., Diederichs, K., Wang, M. \& Caffrey, M. (2016). Acta Cryst. D72, 93-112.

Huang, C.-Y., Olieric, V., Ma, P., Panepucci, E., Diederichs, K., Wang, M. \& Caffrey, M. (2015). Acta Cryst. D71, 1238-1256.

Hunter, M. S. \& Fromme, P. (2011). Methods, 55, 387-404.

Hunter, M. S. et al. (2014). Sci. Rep. 4, 6026.

Kabsch, W. (2010a). Acta Cryst. D66, 133-144.

Kabsch, W. (2010b). Acta Cryst. D66, 125-132.

Kisselman, G., Qiu, W., Romanov, V., Thompson, C. M., Lam, R., Battaile, K. P., Pai, E. F. \& Chirgadze, N. Y. (2011). Acta Cryst. D67, 533-539.

Lamb, A. L., Kappock, T. J. \& Silvaggi, N. R. (2015). Biochim. Biophys. Acta, 1854, 258-268.

Lou, C., Shindel, M., Graham, L. \& Wang, S.-W. (2008). Langmuir, 24, 8111-8118.

Lyubimov, A. Y., Murray, T. D., Koehl, A., Araci, I. E., Uervirojnangkoorn, M., Zeldin, O. B., Cohen, A. E., Soltis, S. M., Baxter, E. L., Brewster, A. S., Sauter, N. K., Brunger, A. T. \& Berger, J. M. (2015). Acta Cryst. D71, 928-940.

Marassi, F. M. \& Opella, S. J. (1998). Curr. Opin. Struct. Biol. 8, 640648.

Martin, R. W. \& Zilm, K. W. (2003). J. Magn. Reson. 165, 162-174.

Matsumura, H., Sugiyama, S., Hirose, M., Kakinouchi, K., Maruyama, M., Murai, R., Adachi, H., Takano, K., Murakami, S., Mori, Y. \& Inoue, T. (2011). J. Synchrotron Rad. 18, 16-19.

McPherson, A. \& DeLucas, L. J. (2015). NPJ Microgravity, 1, 15010.
Moraes, I., Evans, G., Sanchez-Weatherby, J., Newstead, S. \& Stewart, P. D. S. (2014). Biochim. Biophys. Acta, 1838, 78-87.

Murray, T. D., Lyubimov, A. Y., Ogata, C. M., Vo, H., Uervirojnangkoorn, M., Brunger, A. T. \& Berger, J. M. (2015). Acta Cryst. D71, 1987-1997.

Murshudov, G. N., Vagin, A. A. \& Dodson, E. J. (1997). Acta Cryst. D53, 240-255.

Oghbaey, S. et al. (2016). Acta Cryst. D72, 944-955.

Pedrini, B. et al. (2014). Philos. Trans. R. Soc. London Sect. B, 369, 20130500.

Roedig, P., Vartiainen, I., Duman, R., Panneerselvam, S., Stübe, N., Lorbeer, O., Warmer, M., Sutton, G., Stuart, D. I., Weckert, E., David, C., Wagner, A. \& Meents, A. (2015). Sci. Rep. 5, 10451.

Ruprecht, J. \& Nield, J. (2001). Prog. Biophys. Mol. Biol. 75, 121-164.

Sader, K., Studer, D., Zuber, B., Gnaegi, H. \& Trinick, J. (2009). Ultramicroscopy, 110, 43-47.

Schebor, C., Mazzobre, M. F. \& Buera, M. del P. (2010). Carbohydr. Res. 345, 303-308.

Schlichting, I. (2015). IUCrJ, 2, 246-255.

Shimizu, N., Sugiyama, S., Maruyama, M., Yoshikawa, H. Y., Takahashi, Y., Adachi, H., Takano, K., Murakami, S., Inoue, T., Matsumura, H. \& Mori, Y. (2009). Cryst. Growth Des. 9, 52275232.

Sugahara, M. et al. (2016). Sci. Rep. 6, 24484.

Vagin, A. \& Teplyakov, A. (1997). J. Appl. Cryst. 30, 1022-1025.

Vinothkumar, K. R. (2015). Curr. Opin. Struct. Biol. 33, 103-114.

Weierstall, U. et al. (2014). Nat. Commun. 5, 3309.

Wierman, J. L., Alden, J. S., Kim, C. U., McEuen, P. L. \& Gruner, S. M. (2013). J. Appl. Cryst. 46, 1501-1507.

Wlodawer, A., Minor, W., Dauter, Z. \& Jaskolski, M. (2007). FEBS J. 275, $1-21$.

Wüthrich, K. (1990). J. Biol. Chem. 265, 22059-22062.

Zarrine-Afsar, A., Barends, T. R. M., Müller, C., Fuchs, M. R., Lomb, L., Schlichting, I. \& Miller, R. J. D. (2012). Acta Cryst. D68, 321323. 